\title{
АСИМПТОТИКА СПЕКТРА ПЕРИОДИЧЕСКОЙ КРАЕВОЙ ЗАДАЧИ ДЛЯ ДИФФЕРЕНЦИАЛЬНОГО ОПЕРАТОРА С СУММИРУЕМЫМ ПОТЕНЦИАЛОМ
}

\section{С. И. Митрохин}

\begin{abstract}
В статье исследуется спектр дифференциального оператора высокого нечётного порядка с суммируемым потенциалом. Граничные условия являются периодическими. Дифференциальное уравнение, задающее дифференциальный оператор, сводится к интегральному уравнению Вольтерра. Решая это уравнение методом последовательных приближений Пикара, найдена асимптотика фундаментальной системы решений исходного дифференциального уравнения. С помощью этой фундаментальной системы решений изучены периодические граничные условия. В результате выведено уравнение на собственные значения изучаемого дифференциального оператора. Это уравнение представляет собой определитель высокого порядка, который является целой функцией спектрального параметра. Исследована индикаторная диаграмма, соответствующая этой функции. Индикаторная диаграмма является правильным многоугольником и определяет расположение собственных значений рассматриваемого оператора. В результате в каждом из секторов комплексной плоскости, определяемых индикаторной диаграммой, найдена асимптотика собственных значений исследуемого оператора (15 порядка).
\end{abstract}

Ключевые слова: спектральный параметр, дифференциальный оператор, суммируемый потенциал, периодические граничные условия, асимптотика решений дифференциального уравнения, асимптотика спектра.

S. I. Mitrokhin. Asymptotics of the spectrum of a periodic boundary value problem for a differential operator with a summable potential.

The spectrum of a differential operator of a high odd order with summable potential is studied. The boundary conditions are periodic. The differential equation that defines the differential operator is reduced to the Volterra integral equation. Solving this equation by Picard's method of successive approximations, we find the asymptotics of the fundamental system of solutions of the original differential equation. This fundamental system of solutions is used for the study of periodic boundary conditions. As a result, an equation for the eigenvalues of the differential operator is derived. This equation is a determinant of high order, which is an entire function of the spectral parameter. The indicator diagram corresponding to this function is investigated. The indicator diagram is a regular polygon and determines the location of the eigenvalues of the operator under consideration. As a result, the asymptotic behavior of the eigenvalues of the operator is found in each of the sectors of the complex plane determined by the indicator diagram (of 15th order).

Keywords: spectral parameter, differential operator, summable potential, periodic boundary conditions, asymptotics of solutions of a differential equation, asymptotics of the spectrum.

MSC: 47F05, 47A10

DOI: $10.21538 / 0134-4889-2019-25-1-136-149$

\section{1. Постановка задачи}

Изучим спектральные свойства периодической краевой задачи для дифференциального оператора, задаваемого дифференциальным уравнением

$$
y^{(15)}(x)+q(x) y(x)=\lambda a^{15} y(x), \quad 0 \leqslant x \leqslant \pi, \quad a>0,
$$

с краевыми условиями вида

$$
y(0)=y(\pi) ; \quad y^{(m)}(0)=y^{(m)}(\pi), \quad m=1,2, \ldots, 14
$$


Будем предполагать, что функция $q(x)$ является суммируемой по Лебегу на отрезке $[0 ; \pi]$

$$
q(x) \in L_{1}[0 ; \pi] \Leftrightarrow\left(\int_{0}^{x} q(t) d t\right)_{x}^{\prime}=q(x)
$$

почти для всех значений $x$ из отрезка $[0 ; \pi]$.

В уравнении (1.1) число $\lambda \in \mathbb{C}$-спектральный параметр, функция $q(x)$ - потенциал, функция $\rho(x)=a^{15}>0$ - весовая функция.

\section{2. Исторический обзор}

Уже достаточно давно изучаются вопросы спектральной теории дифференциальных операторов, связанных с периодичностью коэффициентов, решений или граничных условий. Свойства периодических решений для оператора второго порядка с гладкими коэффициентами изложены в монографии $[1$, гл. $1, \S 4]$. В классической работе [2] в случае гладкости потенциала изучались дифференциальные операторы второго порядка с периодическими граничными условиями. Исторически сложилось так, что сначала спектральные свойства обыкновенных дифференциальных операторов рассматривались в том случае, когда коэффициенты дифференциальных уравнений, задающих эти операторы, были достаточно гладкими функциями. Асимптотические формулы для корней квазимногочленов, которые возникают при изучении операторов высоких порядков с регулярными граничными условиями (с гладкими коэффициентами), были выведены в работе [3].

Регуляризованные следы такого типа операторов вычислены в статье [4]. В [5] были вычислены регуляризованные следы для дифференциальных операторов четного порядка с разделенными граничными условиями в случае достаточно гладких коэффициентов.

В публикации [6] была изучена обратная задача для квадратичного пучка оператора Штурма - Лиувилля с периодическим потенциалом. Оператор Шредингера, потенциалом которого является тригонометрический полином (т. е. периодическая функция), рассматривался в [7].

В работах $[8 ; 9]$ автору удалось понизить гладкость коэффициентов дифференциальных операторов и были исследованы операторы с кусочно гладкими коэффициентами. Случай дифференциального оператора с кусочно гладкой весовой функцией анализировался автором в [10].

В статьях [11-14] изучались различные спектральные свойства периодических дифференциальных операторов четвертого порядка. Заметим при этом, что с возрастанием порядка дифференциальных операторов сложность выкладок возрастает многократно.

Резкий психологический толчок в изучении дифференциальных операторов с негладкими коэффициентами был сделан совсем недавно, когда в работе [15] были исследованы различные операторы второго порядка с суммируемым потенциалом и вычислены асимптотики произвольного порядка собственных значений и собственных функций таких операторов. Методика этой работы для изучения спектральных свойств операторов с суммируемыми коэффициентами не переносится на операторы четвертого, шестого и более высоких порядков.

Новая методика изучения дифференциальных операторов высоких порядков с суммируемыми коэффициентами разработана автором в работах [16-19]. В [16] был исследован оператор четвертого порядка, у которого не только потенциал, но и коэффициент при первой производной были суммируемыми функциями на отрезке задания оператора. В [17] изучен дифференциальный оператор произвольного нечетного порядка со стандартными разделенными граничными условиями.

В статье [18] исследован дифференциальный оператор высокого четного порядка с разделенными граничными условиями, зависящими от некоторых параметров. В [19] был изучен оператор шестого порядка с запаздывающим аргументом с суммируемыми коэффициентами 
на отрезке (граничные условия разделенные) и найдена асимптотика собственных значений и собственных функций.

Во всех этих исследованиях граничные условия были разделенными; неразделенные граничные условия для операторов порядка выше второго фактически не изучались. В работе [20] был изучен дифференциальный оператор четвертого порядка с периодическими граничными условиями с суммируемым потенциалом. В результате сложных технических выкладок, связанных с четностью порядка оператора, было доказано, что спектр оператора является дискретным и была найдена асимптотика собственных значений этого оператора, с помощью которой можно изучить асимптотику собственных функций.

\section{3. Асимптотика решений дифференциального уравнения (1.1) при $\lambda \rightarrow \infty$}

Введем следующие обозначения: $\lambda=s^{15}, s=\sqrt[15]{\lambda}$, при этом зафиксируем ту ветвь арифметического корня, для которой $\sqrt[15]{1}=+1$. Пусть $w_{k}(k=1,2, \ldots, 15)$-различные корни пятнадцатой степени из единицы:

$$
\begin{gathered}
w_{k}^{15}=1, \quad w_{k}=e^{\frac{2 \pi i}{15}(k-1)}, \quad k=1,2, \ldots, 15 ; \quad w_{1}=1, \quad w_{2}=e^{\frac{2 \pi i}{15}}=\theta \neq 0 \\
w_{3}=e^{\frac{4 \pi i}{15}}=\theta^{2} ; \quad \ldots ; \quad w_{m}=\theta^{m-1}, \quad m=1,2, \ldots, 15 ; \quad w_{m+15}=w_{m} .
\end{gathered}
$$

Числа $w_{k}(k=1,2, \ldots, 15)$ из $(3.1)$ удовлетворяют следующим свойствам:

$$
\sum_{k=1}^{15} w_{k}^{n}=0, n=1,2, \ldots, 14 ; \quad \sum_{k=1}^{15} w_{k}^{n}=15, n=0, n=15 .
$$

Аналогично работам $[16 ; 17 ; 21$, гл. 2] устанавливается следующее утверждение.

Теорема 1. Общее решение дифференииального уравнения (1.1) представляется в следующем виде:

$$
y(x, s)=\sum_{k=1}^{15} C_{k} y_{k}(x, s) ; \quad y^{(m)}(x, s)=\sum_{k=1}^{15} C_{k} y_{k}^{(m)}(x, s), \quad m=1,2, \ldots, 14,
$$

где $C_{k}(k=1,2, \ldots, 15)$ - произвольные постояннъе, при этом для фундаментальной системы решений $\left\{y_{k}(x, s)\right\}_{k=1}^{15}$ справедливы следующие асимптотические формулы и оценки:

$$
\begin{gathered}
y_{k}(x, s)=e^{a w_{k} s x}-\frac{A_{14, k}(x, s)}{15 a^{14} s^{14}}+\underline{O}\left(\frac{e^{|\operatorname{Im} s| a x}}{s^{28}}\right), \quad k=1,2, \ldots, 15 \\
y_{k}^{(m)}(x, s)=(a s)^{m}\left\{w_{k}^{m} e^{a w_{k} s x}-\frac{A_{14, k}^{m}(x, s)}{15 a^{14} s^{14}}+\underline{O}\left(\frac{e^{|\operatorname{Im} s| a x}}{s^{28}}\right)\right\}, \quad k=1,2, \ldots, 15 ; \quad m=1,2, \ldots, 14 \\
A_{14, k}(x, s)=\sum_{k=1}^{15} w_{n} e^{a w_{n} s x} \int_{0}^{x} q(t) e^{a\left(w_{k}-w_{n}\right) s t} d t_{a k n}, \quad k=1,2, \ldots, 15 \\
\left.A_{14, k}^{m}(x, s)=\sum_{n=1}^{15} w_{n} w_{n}^{m} e^{a w_{n} s x}\left(\int_{0}^{x} \ldots\right)_{a k n}, \quad k=1,2, \ldots, 15 ; \quad m=1,2, \ldots, 14.5\right)
\end{gathered}
$$

При выводе формул (3.4)-(3.7) мы требовали выполнения следующих начальных условий:

$$
\begin{gathered}
A_{14, k}(0, s)=0 ; \quad A_{14, k}^{m}(0, s)=0 ; \quad y_{k}(0, s)=1 ; \quad y_{k}^{(m)}(0, s)=w_{n}^{m}(a s)^{m}, \\
k=1,2, \ldots, 15 ; \quad m=1,2, \ldots, 14 .
\end{gathered}
$$




\section{4. Изучение граничных условий (1.2)}

Подставляя формулы (3.3) в граничные условия (1.2), получаем

$$
\left\{\begin{array}{l}
y(\pi, s)=y(0, s) \Leftrightarrow \sum_{k=1}^{15} C_{k} y_{k}(\pi, s) \\
=\sum_{k=1}^{15} C_{k} y_{k}(0, s) \Leftrightarrow \sum_{k=1}^{15} C_{k}\left[y_{k}(\pi, s)-y_{k}(0, s)\right]=0, \quad y_{k}(0, s) \stackrel{(3.8)}{=} 1 \\
y^{(m)}(\pi, s)=y^{(m)}(0, s) \Leftrightarrow \sum_{k=1}^{15} C_{k} y_{k}^{(m)}(\pi, s) \\
=\sum_{k=1}^{15} C_{k} y_{k}^{(m)}(0, s) \Leftrightarrow \sum_{k=1}^{15} C_{k}\left[\frac{y_{k}^{(m)}(\pi, s)}{(a s)^{m}}-\frac{y_{k}^{(m)}(0, s)}{(a s)^{m}}\right]=0 \\
\frac{y_{k}^{(m)}(0, s)}{(a s)^{m}}=w_{k}^{m}, \quad m=1,2, \ldots, 14 .
\end{array}\right.
$$

Система (4.1), (4.2) представляет собой однородную систему из пятнадцати линейных уравнений с пятнадцатью неизвестными $C_{1}, C_{2}, \ldots, C_{15}$. Из метода Крамера следует, что такая система имеет ненулевые решения только в том случае, когда ее определитель равен нулю. Поэтому верна следующая теорема.

Теорема 2. Уравнение на собственные значения периодической краевой задачи (1.1), (1.2) с условием (1.3) суммируемости потенииала представляется в следующем виде:

$$
f(s)=\left|\begin{array}{crrr}
y_{1}(\pi, s)-y_{1}(0, s) & y_{2}(\pi, s)-y_{2}(0, s) & \ldots & y_{15}(\pi, s)-y_{15}(0, s) \\
\frac{y_{1}^{\prime}(\pi, s)}{a s}-\frac{y_{1}^{\prime}(0, s)}{a s} & \frac{y_{2}^{\prime}(\pi, s)}{a s}-\frac{y_{2}^{\prime}(0, s)}{a s} & \ldots & \frac{y_{15}^{\prime}(\pi, s)}{a s}-\frac{y_{15}^{\prime}(0, s)}{a s} \\
\frac{y_{1}^{\prime \prime}(\pi, s)}{(a s)^{2}}-\frac{y_{1}^{\prime \prime}(0, s)}{(a s)^{2}} & \frac{y_{2}^{\prime \prime}(\pi, s)}{(a s)^{2}}-\frac{y_{2}^{\prime \prime}(0, s)}{(a s)^{2}} & \ldots & \frac{y_{15}^{\prime \prime}(\pi, s)}{(a s)^{2}}-\frac{y_{15}^{\prime \prime}(0, s)}{(a s)^{2}} \\
\ldots \ldots \ldots \ldots \ldots \ldots \\
\frac{y_{1}^{(14)}(\pi, s)}{(a s)^{14}}-\frac{y_{1}^{(14)}(0, s)}{(a s)^{14}} & \frac{y_{2}^{(14)}(\pi, s)}{(a s)^{14}}-\frac{y_{2}^{(14)}(0, s)}{(a s)^{14}} & \ldots & \frac{y_{15}^{(14)}(\pi, s)}{(a s)^{14}}-\frac{y_{15}^{(14)}(0, s)}{(a s)^{14}}
\end{array}\right|=0 .
$$

Используя формулы (3.4), (3.5) и начальные условия (3.8), уравнение (4.3) можно переписать в следующем виде, введя обозначение $h_{m}=e^{a w_{m} s x}, m=1,2, \ldots, 15$ :

$$
\begin{aligned}
& f(s)=\left|\begin{array}{ccc}
{\left[h_{1}-1\right]-\frac{A_{14,1}(\pi, s)}{15 a^{14} s^{14}}+\ldots} & \ldots & {\left[h_{15}-1\right]-\frac{A_{14,15}(\pi, s)}{15 a^{14} s^{14}}+\ldots} \\
w_{1}\left[h_{1}-1\right]-\frac{A_{14,1}^{1}(\pi, s)}{15 a^{14} s^{14}}+\ldots & \ldots & w_{15}\left[h_{15}-1\right]-\frac{A_{14,15}^{1}(\pi, s)}{15 a^{14} s^{14}}+\ldots \\
w_{1}^{2}\left[h_{1}-1\right]-\frac{A_{14,1}^{2}(\pi, s)}{15 a^{14} s^{14}}+\ldots & \ldots & w_{15}^{2}\left[h_{15}-1\right]-\frac{A_{14,15}^{2}(\pi, s)}{15 a^{14} s^{14}}+\ldots \\
\ldots \ldots \ldots \ldots & \ldots \ldots \\
\ldots \ldots & \ldots \ldots & \ldots \ldots \\
w_{1}^{14}\left[h_{1}-1\right]-\frac{A_{14,1}^{14}(\pi, s)}{15 a^{14} s^{14}}+\ldots & \ldots & w_{15}^{14}\left[h_{15}-1\right]-\frac{A_{14,15}^{14}(\pi, s)}{15 a^{14} s^{14}}+\ldots
\end{array}\right|=0, \\
& 《+\ldots »=+\underline{O}\left(\frac{1}{s^{28}}\right) .
\end{aligned}
$$

Раскладывая определитель $f(s)$ из (4.4) на сумму определителей по столбцам, имеем

$$
f(s)=f_{0}(s)-\frac{f_{14}(s)}{15 a^{14} s^{14}}+\underline{O}\left(\frac{1}{s^{28}}\right)=0,
$$




$$
\begin{aligned}
& f_{0}(s)=\left|\begin{array}{lrrrr}
1 \cdot\left[h_{1}-1\right] & 1 \cdot\left[h_{2}-1\right] & \ldots & 1 \cdot\left[h_{14}-1\right] & 1 \cdot\left[h_{15}-1\right] \\
w_{1}\left[h_{1}-1\right] & w_{2}\left[h_{2}-1\right] & \ldots & w_{14}\left[h_{14}-1\right] & w_{15}\left[h_{15}-1\right] \\
\ldots \ldots \ldots & \ldots \ldots \ldots \ldots & \ldots & \ldots \ldots \ldots \ldots \ldots & \ldots \ldots \ldots \\
w_{1}^{14}\left[h_{1}-1\right] & w_{2}^{14}\left[h_{2}-1\right] & \ldots & w_{14}^{14}\left[h_{14}-1\right] & w_{15}^{14}\left[h_{15}-1\right]
\end{array}\right| \\
& =\Delta_{00}\left[h_{1}-1\right]\left[h_{2}-1\right](\ldots)\left[h_{14}-1\right]\left[h_{15}-1\right], \quad \Delta_{00} \neq 0 \text {, } \\
& \Delta_{00}=\left|\begin{array}{ccccc}
1 & 1 & \ldots & 1 & 1 \\
w_{1} & w_{2} & \ldots & w_{14} & w_{15} \\
\ldots \ldots & \ldots & \ldots & \ldots & \ldots \\
w_{1}^{14} & w_{2}^{14} & \ldots & w_{14}^{14} & w_{15}^{14}
\end{array}\right| \\
& =\operatorname{det} \text { Wandermoun's }\left(w_{1}, w_{2}, \ldots, w_{14}, w_{15}\right)=\prod_{\substack{k>n \\
k, n=1,2, \ldots, 15}}\left(w_{k}-w_{n}\right) \neq 0 \text {; } \\
& f_{14}(s)=f_{14,1}(s)+f_{14,2}(s)+\cdots+f_{14,14}(s)+f_{14,15}(s), \\
& f_{14,1}(s)=\left|\begin{array}{lllll}
A_{14,1}(\pi, s) & 1 \cdot\left[h_{2}-1\right] & \ldots & 1 \cdot\left[h_{14}-1\right] & 1 \cdot\left[h_{15}-1\right] \\
A_{14,1}^{1}(\pi, s) & w_{2}\left[h_{2}-1\right] & \ldots & w_{14}\left[h_{14}-1\right] & w_{15}\left[h_{15}-1\right] \\
\ldots \ldots \ldots \ldots & \ldots \ldots \ldots \ldots & \ldots & \ldots \ldots \ldots \ldots \ldots & \ldots \ldots \ldots \ldots \\
A_{14,1}^{14}(\pi, s) & w_{2}^{14}\left[h_{2}-1\right] & \ldots & w_{14}^{14}\left[h_{14}-1\right] & w_{15}^{14}\left[h_{15}-1\right]
\end{array}\right|
\end{aligned}
$$

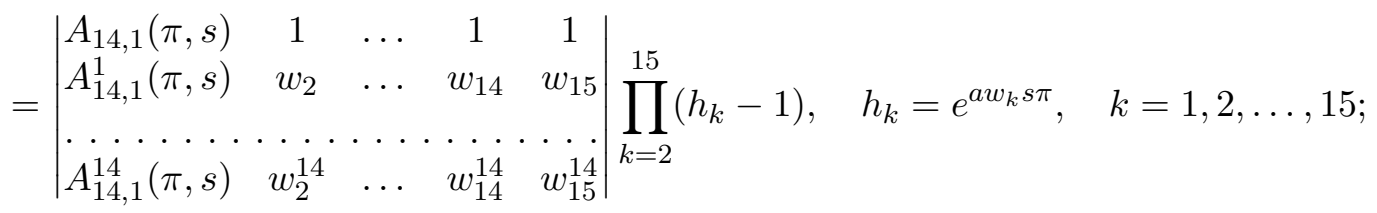

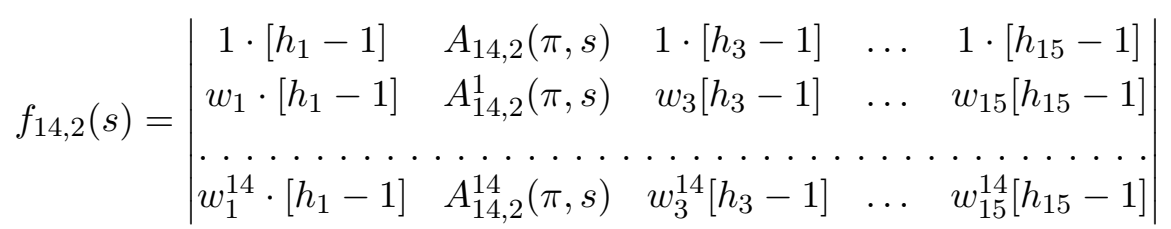

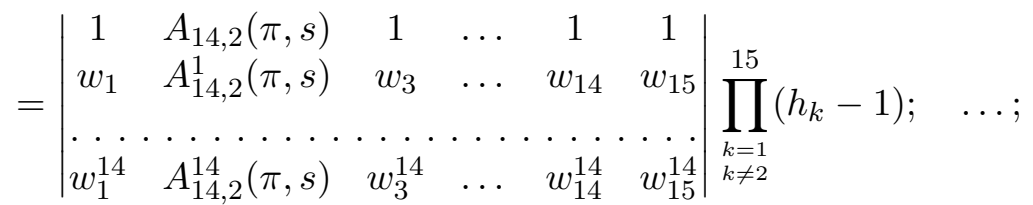

$$
\begin{aligned}
& f_{14,14}(s)=\left|\begin{array}{cccccc}
1 & 1 & \ldots & 1 & A_{14,14}(\pi, s) & 1 \\
w_{1} & w_{2} & \ldots & w_{13} & A_{14,14}^{1}(\pi, s) & w_{15} \\
\ldots & \ldots & \ldots & \ldots \ldots \ldots \ldots \ldots \ldots \ldots \ldots & \ldots & \ldots \\
w_{1}^{14} & w_{2}^{14} & \ldots & w_{13}^{14} & A_{14,14}^{14}(\pi, s) & w_{15}^{14}
\end{array}\right| \prod_{k=1}^{15}\left(h_{k}-1\right) \\
& f_{14,15}(s)=\left|\begin{array}{cccccc}
1 & 1 & \ldots & 1 & 1 & A_{14,15}(\pi, s) \\
w_{1} & w_{2} & \ldots & w_{13} & w_{14} & A_{14,15}^{1}(\pi, s) \\
\ldots \ldots & \ldots & \ldots & \ldots & \ldots \ldots & \ldots \ldots \ldots \ldots \\
w_{1}^{14} & w_{2}^{14} & \ldots & w_{13}^{14} & w_{14}^{14} & A_{14,15}^{14}(\pi, s)
\end{array}\right| \prod_{k=1}^{14}\left(h_{k}-1\right) .
\end{aligned}
$$

Основное приближение уравнения (4.5)-(4.12) имеет вид

$$
f_{0}(s)=0
$$

где функция $f_{0}(s)$ определена формулами (4.6), (4.7). 


\section{5. Изучение основного приближения. Индикаторная диаграмма}

Из формулы (4.6) имеем

$$
\begin{gathered}
f_{0}(s)=\Delta_{00}\left(e^{a w_{1} s \pi}-1\right)\left(e^{a w_{2} s \pi}-1\right)\left(e^{a w_{3} s \pi}-1\right)(\ldots)\left(e^{a w_{14} s \pi}-1\right)\left(e^{a w_{15} s \pi}-1\right) \\
=\Delta_{00}\left(e^{a\left(w_{1}+w_{2}\right) s \pi}-e^{a w_{1} s \pi}-e^{a w_{2} s \pi}+1\right)\left(e^{a w_{3} s \pi}-1\right)(\ldots)\left(e^{a w_{14} s \pi}-1\right)\left(e^{a w_{15} s \pi}-1\right) \\
=\Delta_{00}\left(e^{a\left(w_{1}+w_{2}+w_{3}\right) s \pi}-e^{a\left(w_{1}+w_{2}\right) s \pi}-e^{a\left(w_{1}+w_{3}\right) s \pi}-e^{a\left(w_{2}+w_{3}\right) s \pi}\right. \\
\left.+e^{a w_{1} s \pi}+e^{a w_{2} s \pi}+e^{a w_{3} s \pi}-1\right)(\ldots)\left(e^{a w_{14} s \pi}-1\right)\left(e^{a w_{15} s \pi}-1\right)=\ldots \\
=\Delta_{00}\left\{-1+\sum_{k=1}^{15} e^{a w_{k} s \pi}-\sum_{\substack{k, n=1 \\
k \neq n}}^{15} e^{a\left(w_{k}+w_{n}\right) s \pi}+\sum_{\substack{k, n, m=1 \\
k \neq n ; k \neq m ; n \neq m}}^{15} e^{a\left(w_{k}+w_{n}+w_{m}\right) s \pi}+\sum_{p=4}^{15}(-1)^{p-1} \phi_{p}\right\} \\
\phi_{p}=\sum \exp \left(\sum_{r=1}^{p} w_{k_{r}}\right), \quad p=1,2,3, \ldots, 15 .
\end{gathered}
$$

Для исследования корней уравнения (4.13) с учетом (5.1) необходимо изучить так называемую индикаторную диаграмму (см. [22, гл. 12]), т. е. выпуклую оболочку показателей экспонент, входящих в это уравнение. В результате исследования этого множества точек приходим к выводу, что индикаторная диаграмма уравнения (4.13), (5.1) имеет следующий вид (см. рисунок).

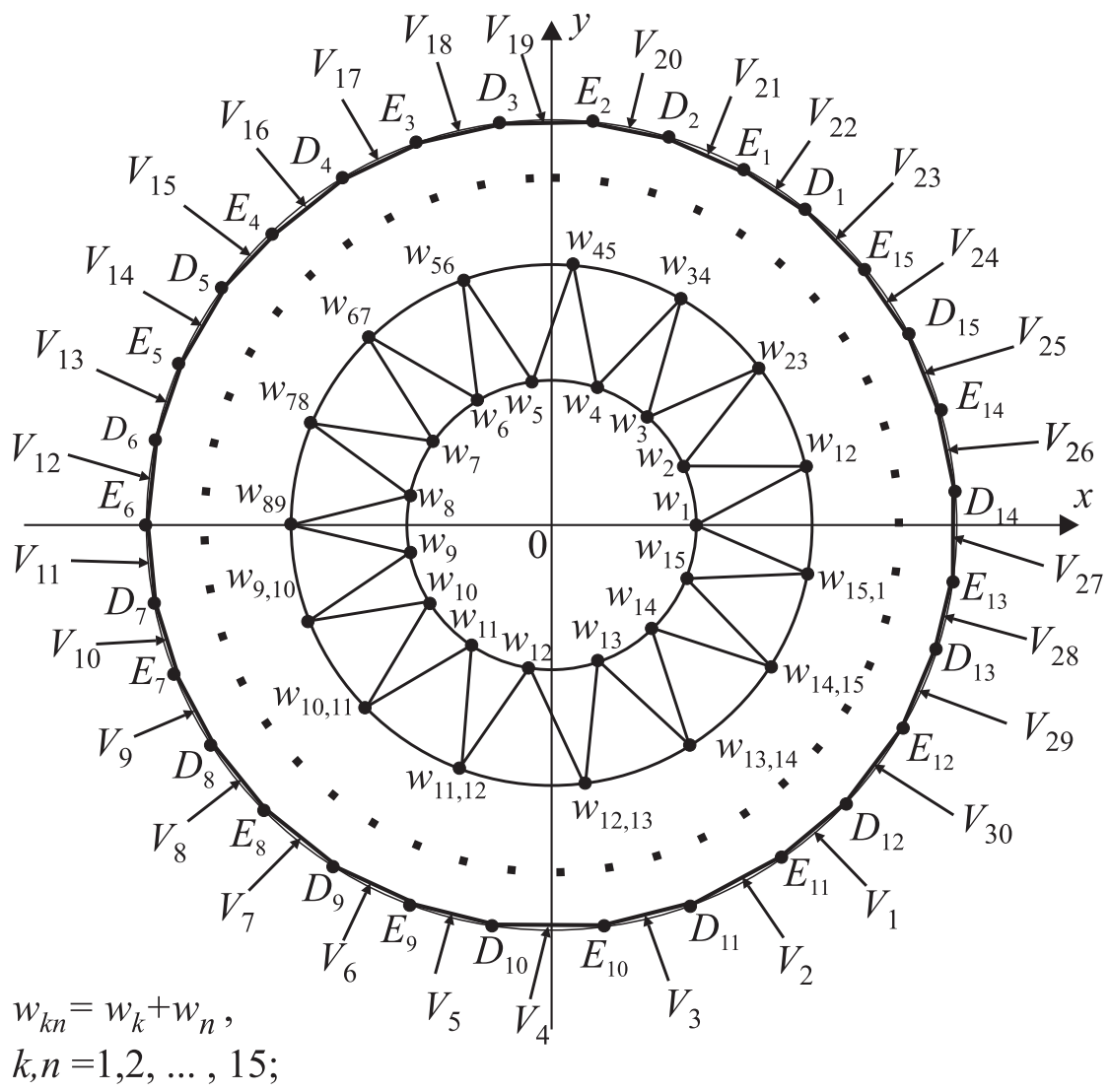

Действительно, на внутренней окружности радиуса 1 расположены точки, соответствующие сумме $\sum_{k=1}^{15} e^{a w_{k} s \pi}$, т. е. точки $w_{k}(k=1,2, \ldots, 15)$, они делят единичную окружность на пятнадцать равных частей. Но в силу свойства (3.2) на этой же окружности находятся точки, соответствующие сумме $\phi_{14}=\sum \exp \left(\sum_{r=1}^{14} w_{k_{r}}\right)$, т. е. точки

$w_{1}+w_{2}+\cdots+w_{14}=-w_{15}, \quad w_{2}+w_{3}+\cdots+w_{14}+w_{15}=-w_{1}, \quad w_{1}+w_{3}+w_{4}+\cdots+w_{15}=-w_{2}$ 
и т. д., иначе говоря, точки $-w_{1},-w_{2}, \ldots,-w_{14},-w_{15}$ также делят единичную окружность на пятнадцать равных частей, но не совпадают с точками $w_{1}, w_{2}, \ldots, w_{15}$; точки $-w_{1},-w_{2}, \ldots$, $-w_{14},-w_{15}$ в силу нечетности их количества являются серединами дуг

$$
w_{8} w_{9}, \quad w_{9} w_{10}, \quad \ldots, \quad w_{14} w_{15}, \quad w_{15} w_{1}, \quad w_{1} w_{2}, \quad \ldots, \quad w_{7} w_{8} .
$$

Точки, соответствующие сумме $\sum_{\substack{k, n=1 \\ k \neq n}}^{15} e^{a\left(w_{k}+w_{n}\right) s \pi}$ из $(5.1)$, т. е. точки $w_{1}+w_{2}, w_{1}+w_{3}$, $w_{1}+w_{4}, w_{2}+w_{3}, w_{2}+w_{4}, \ldots$, построим по правилу параллелограмма сложения векторов. В результате получим, что выпуклой оболочкой этого множества точек являются точки

$$
w_{1}+w_{2}, \quad w_{2}+w_{3}, w_{3}+w_{4}, \ldots, w_{14}+w_{15}, \quad w_{15}+w_{1},
$$

они лежат на окружности радиуса $R_{2}=\left|w_{1}+w_{2}\right|>1$ и делят эту окружность на пятнадцать равных частей. Точки вида $w_{1}+w_{3}, w_{1}+w_{4}, w_{2}+w_{4}, \ldots, w_{k}+w_{n}$ при условии $|k-n| \geqslant 2$ попадают внутрь этой окружности и на асимптотику корней уравнения (4.13), (5.1) не влияют (см. [22, гл. 2]). На этой же окружности находятся точки, соответствующие сумме $\phi_{13}=\sum \exp \left(\sum_{r=1}^{13} w_{k_{r}}\right)$ из $(5.1)$; выпуклую оболочку этого множества точек образуют точки

$$
\begin{gathered}
-w_{1}-w_{2}=w_{3}+w_{4}+\cdots+w_{15}, \quad-w_{2}-w_{3}=w_{1}+w_{4}+\cdots+w_{15}, \\
-w_{3}-w_{4}=w_{5}+\cdots+w_{15}+w_{1}+w_{2}, \quad \ldots,
\end{gathered}
$$

они делят окружность радиуса $R_{2}=\left|w_{1}+w_{2}\right|$ на пятнадцать равных частей и являются серединами дуг

$$
\begin{gathered}
{\left[w_{8}+w_{9} ; w_{9}+w_{10}\right], \quad\left[w_{8}+w_{10} ; w_{10}+w_{11}\right], \quad \ldots} \\
{\left[w_{14}+w_{15} ; w_{15}+w_{1}\right], \quad\left[w_{15}+w_{1} ; w_{1}+w_{2}\right], \quad \ldots, \quad\left[w_{7}+w_{8} ; w_{8}+w_{9}\right] .}
\end{gathered}
$$

Точки вида $-w_{1}-w_{3},-w_{1}-w_{4},-w_{2}-w_{4}, \ldots,-w_{k}-w_{p}$ при условии $|k-p| \geqslant 2$ попадают внутрь этой окружности и на асимптотику корней уравнения (4.13), (5.1) не влияют.

Аналогичным образом устанавливаем, что выпуклую оболочку множеств точек, соответствующих суммам

$$
\sum_{\substack{k, n, m=1 \\ k \neq n, k \neq m, n \neq m}}^{15} e^{a\left(w_{k}+w_{n}+w_{m}\right) s \pi} \text { и } \phi_{12}=\sum \exp \left(\sum_{r=1}^{12} w_{k_{r}}\right) \text { из (5.1), }
$$

образуют точки

$$
\begin{aligned}
& \pm\left(w_{1}+w_{2}+w_{3}\right), \pm\left(w_{2}+w_{3}+w_{4}\right), \quad \pm\left(w_{3}+w_{4}+w_{5}\right), \ldots, \quad \pm\left(w_{13}+w_{14}+w_{15}\right) \\
& \pm\left(w_{14}+w_{15}+w_{16}\right)= \pm\left(w_{14}+w_{15}+w_{1}\right), \quad \pm\left(w_{15}+w_{16}+w_{17}\right)= \pm\left(w_{15}+w_{1}+w_{2}\right)
\end{aligned}
$$

они лежат на окружности радиуса $R_{3}=\left|w_{1}+w_{2}+w_{3}\right|>R_{2}$ и делят эту окружность на тридцать равных частей.

Методом математической индукции доказываем, что выпуклую оболочку множества точек, соответствующих суммам

$$
\phi_{4}=\sum \exp \left(\sum_{r=1}^{4} w_{k_{r}}\right) \text { и } \phi_{11}=\sum \exp \left(\sum_{r=1}^{11} w_{k_{r}}\right) \text { из (5.1), }
$$

образуют точки $\pm\left(w_{1}+w_{2}+w_{3}+w_{4}\right), \pm\left(w_{2}+w_{3}+w_{4}+w_{5}\right), \pm\left(w_{3}+w_{4}+w_{5}+w_{6}\right), \ldots$, они лежат на окружности радиуса $R_{4}=\left|w_{1}+w_{2}+w_{3}+w_{4}\right|>R_{3}$ и делят эту окружность на тридцать равных частей ;...; выпуклую оболочку множества точек, соответствующих суммам

$$
\phi_{7}=\sum \exp \left(\sum_{r=1}^{7} w_{k_{r}}\right) \text { и } \phi_{8}=\sum \exp \left(\sum_{r=1}^{8} w_{k_{r}}\right) \text { из }
$$


образуют точки

$$
D_{1}=\sum_{k=1}^{7} w_{k}, \quad D_{2}=\sum_{k=2}^{8} w_{k}, \quad D_{3}=\sum_{k=3}^{9} w_{k}, \ldots,
$$

они делят окружность радиуса

$$
\begin{gathered}
R_{7}=\left|w_{1}+w_{2}+\cdots+w_{6}+w_{7}\right|>R_{6} \\
=\left|w_{1}+w_{2}+\cdots+w_{6}\right|>R_{5}=\left|w_{1}+w_{2}+\cdots+w_{5}\right|>R_{4}=\left|w_{1}+w_{2}+w_{3}+w_{4}\right|>R_{3}
\end{gathered}
$$

на пятнадцать равных частей, и точки

$$
-\sum_{k=1}^{7} w_{k}, \quad-\sum_{k=2}^{8} w_{k}, \quad-\sum_{k=3}^{9} w_{k}, \ldots,
$$

они также делят окружность радиуса $R_{7}=\left|w_{1}+w_{2}+\cdots+w_{6}+w_{7}\right| \stackrel{(3.2)}{=}\left|w_{1}+w_{2}+\cdots+w_{6}+w_{7}+w_{8}\right|$ на пятнадцать равных частей, при этом эти точки являются серединами дуг

$$
\breve{D_{1} D_{2}}, \quad \widetilde{D_{2} D_{3}}, \quad \widetilde{D_{3} D_{4}}, \quad \ldots, \quad D_{14} D_{15}, \quad \widetilde{D_{15} D_{1}} \text {. }
$$

Из (5.2) следует, что асимптотика корней уравнений (4.13), (5.1) и (4.5)-(4.12) находится в секторах $V_{1}, V_{2}, V_{3}, \ldots, V_{30}$ бесконечно малого раствора, биссектрисы которых являются серединными перпендикулярами к дугам

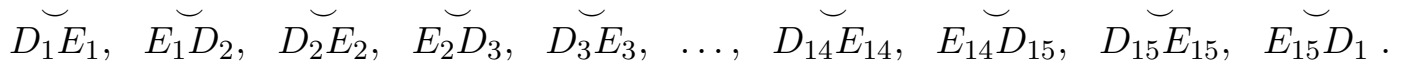

\section{6. Асимптотика собственных значений оператора (1.1)-(1.3) в секторе $V_{1}$}

Из общей теории нахождения асимптотики корней квазиполиномов вида (4.5)-(4.12) следует, что для нахождения корней в секторе $V_{1}$ в уравнении (4.5)-(4.12) (а также (4.13), (5.1)) необходимо оставить только экспоненты, соответствующие точкам $D_{1}$ и $E_{1}$, т. е. надо оставить экспоненты с показателями $w_{1}+w_{2}+\cdots+w_{7}$ и $w_{1}+w_{2}+\cdots+w_{7}+w_{8}$. Очевидно при этом, что у функции $f_{0}(s)$ из (5.1) надо оставить экспоненты

$$
\Delta_{00} \exp \left(a\left(w_{1}+w_{2}+\cdots+w_{7}\right) s \pi\right) \text { и }(-1) \Delta_{00} \exp \left(a\left(w_{1}+w_{2}+\cdots+w_{7}+w_{8}\right) s \pi\right) .
$$

Изучим поведение величин $f_{14,1}(s), f_{14,2}(s), \ldots, f_{14,15}(s)$ из (4.9)-(4.12). Применяя формулы (3.6), (3.7), из формулы (4.9) получаем

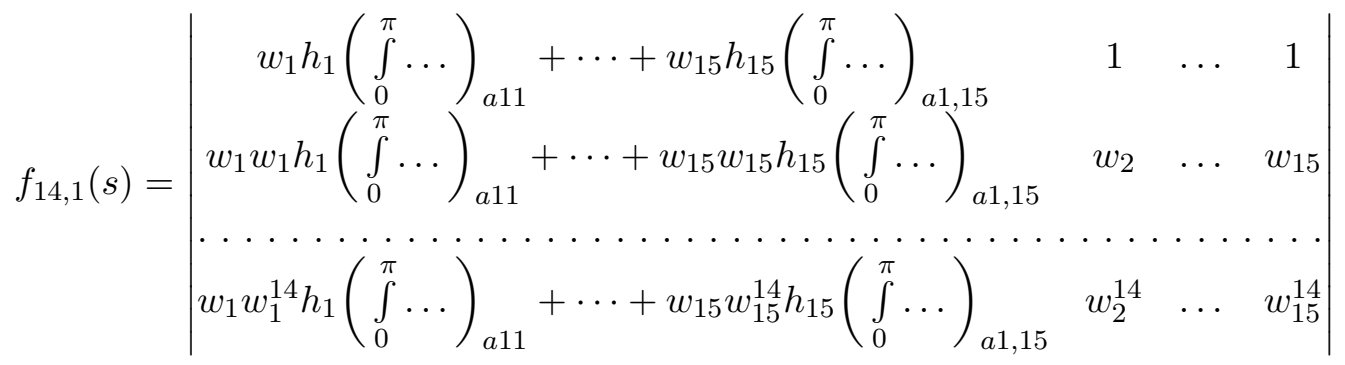

$$
\begin{aligned}
& \times \prod_{k=2}^{15}\left(h_{k}-1\right)=\left\{w_{1} h_{1}\left(\int_{0}^{\pi} \ldots\right)_{a 11} \Delta_{00}+w_{2} h_{2}\left(\int_{0}^{\pi} \ldots\right)_{a 12} \cdot 0\right. \\
& \left.+w_{3} h_{3}\left(\int_{0}^{\pi} \ldots\right)_{a 13} \cdot 0+\cdots+w_{15} h_{15}\left(\int_{0}^{\pi} \ldots\right)_{a 15}\right\} \prod_{k=2}^{15}\left(h_{k}-1\right) \\
& =w_{1} \Delta_{00} e^{a w_{1} s \pi} \int_{0}^{\pi} q(t) d t_{a 11} \prod_{k=2}^{15}\left(e^{a w_{k} s \pi}-1\right),
\end{aligned}
$$




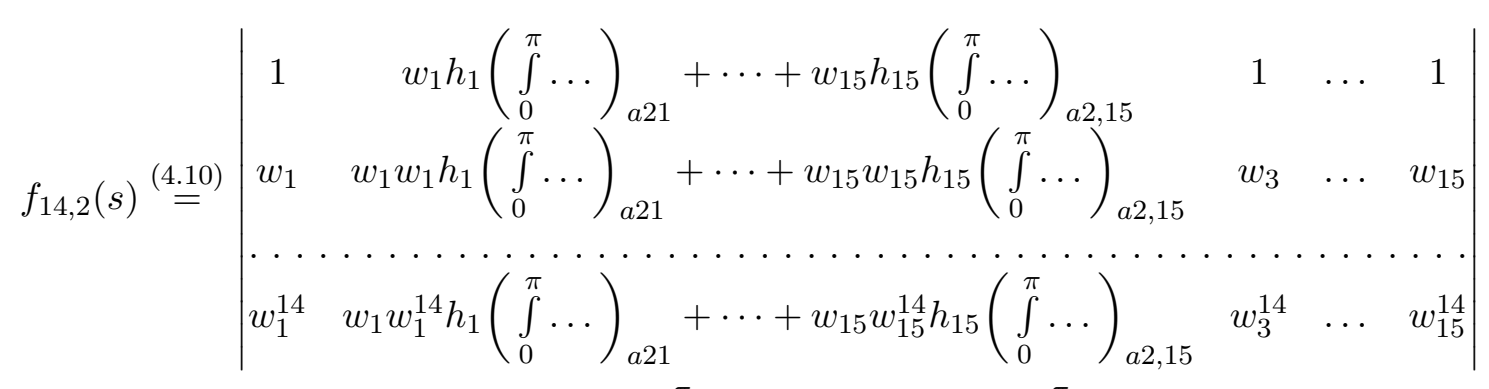

$$
\begin{aligned}
& \times \prod_{\substack{k=1 \\
k \neq 2}}^{15}\left(h_{k}-1\right)=\left\{w_{1} h_{1}\left(\int_{0}^{\pi} \ldots\right)_{a 21} \cdot 0+w_{2} h_{2}\left(\int_{0}^{\pi} \ldots\right)_{a 22} \Delta_{00}\right. \\
& \left.+w_{3} h_{3}\left(\int_{0}^{\pi} \ldots\right)_{a 23} \cdot 0+\cdots+w_{15} h_{15}\left(\int_{0}^{\pi} \ldots\right)_{a 15}\right\} \prod_{\substack{k=1 \\
k \neq 2}}^{15}\left(h_{k}-1\right) \\
& =w_{2} \Delta_{00} e^{a w_{2} s \pi} \int_{0}^{\pi} q(t) d t_{a 22} \prod_{\substack{k=1 \\
k \neq 2}}^{15}\left(e^{a w_{k} s \pi}-1\right) .
\end{aligned}
$$

Аналогичным образом из формул (4.9)-(4.12), (3.6), (3.7) и свойств определителей выводим

$$
\begin{gathered}
f_{14,3}(s)=w_{3} \Delta_{00} \int_{0}^{\pi} q(t) d t_{a 33} e^{a w_{3} s \pi} \prod_{\substack{k=1 \\
k \neq 3}}^{15}\left(e^{a w_{k} s \pi}-1\right) ; \ldots ; \\
f_{14, n}(s)=w_{n} \Delta_{00} \int_{0}^{\pi} q(t) d t_{a n n} e^{a w_{n} s \pi} \prod_{\substack{k=1 \\
k \neq n}}^{15}\left(e^{a w_{k} s \pi}-1\right), \quad n=1,2, \ldots, 15 .
\end{gathered}
$$

С помощью формул (6.1)-(6.3) у функций $f_{14, n}(s)(n=1,2, \ldots, 15)$ можно отобрать экспоненты, нужные для сектора $V_{1}$. Из (6.11) имеем

$$
\begin{gathered}
e^{a w_{1} s \pi} \prod_{k=2}^{15}\left(e^{a w_{k} s \pi}-1\right) \\
=e^{a w_{1} s \pi}\left(e^{a w_{2} s \pi}-1\right)\left(e^{a w_{3} s \pi}-1\right)(\ldots)\left(e^{a w_{7} s \pi}-1\right)\left(e^{a w_{8} s \pi}-1\right)\left(e^{a w_{9} s \pi}-1\right)(\ldots)\left(e^{a w_{15} s \pi}-1\right) \\
=\exp \left(a\left(w_{1}+w_{2}+\cdots+w_{7}\right) s \pi\right)(-1)^{15-7}+\exp \left(a\left(w_{1}+w_{2}+\cdots+w_{7}+w_{8}\right) s \pi\right)(-1)^{15-8}+\ldots,
\end{gathered}
$$

поэтому у функции $f_{14,1}(s)$ из (6.1) для сектора $V_{1}$ надо оставить следующие экспоненты:

$$
w_{1} \Delta_{00} \int_{0}^{\pi} q(t) d t_{a 11}\left[\exp \left(a\left(w_{1}+w_{2}+\cdots+w_{7}\right) s \pi\right)-\exp \left(a\left(w_{1}+w_{2}+\cdots+w_{7}+w_{8}\right) s \pi\right)\right] .
$$

Из формулы (6.2) имеем

$$
\begin{gathered}
e^{a w_{2} s \pi} \prod_{\substack{k=1 \\
k \neq 2}}^{15}\left(e^{a w_{k} s \pi}-1\right) \\
=e^{a w_{2} s \pi}\left(e^{a w_{1} s \pi}-1\right)\left(e^{a w_{3} s \pi}-1\right)(\ldots)\left(e^{a w_{7} s \pi}-1\right)\left(e^{a w_{8} s \pi}-1\right)\left(e^{a w_{9} s \pi}-1\right)(\ldots)\left(e^{a w_{15} s \pi}-1\right) \\
=\exp \left(a\left(w_{1}+w_{2}+\cdots+w_{7}\right) s \pi\right)(-1)^{15-7}+\exp \left(a\left(w_{1}+w_{2}+\cdots+w_{7}+w_{8}\right) s \pi\right)(-1)^{15-8}+\ldots,
\end{gathered}
$$

поэтому у функции $f_{14,2}(s)$ из $(6.2)$ для сектора $V_{1}$ надо оставить следующие экспоненты:

$$
w_{2} \Delta_{00} \int_{0}^{\pi} q(t) d t_{a 22}\left[\exp \left(a\left(w_{1}+w_{2}+\cdots+w_{7}\right) s \pi\right)-\exp \left(a\left(w_{1}+w_{2}+\cdots+w_{7}+w_{8}\right) s \pi\right)\right] .
$$


Аналогичным образом устанавливаем: для функций $f_{14, n}(s)(n=3,4,5,6,7)$ для сектора $V_{1}$ надо оставить экспоненты

$$
w_{n} \Delta_{00} \int_{0}^{\pi} q(t) d t_{a n n}\left[\exp \left(a\left(w_{1}+w_{2}+\cdots+w_{7}\right) s \pi\right)-\exp \left(a\left(w_{1}+w_{2}+\cdots+w_{7}+w_{8}\right) s \pi\right)\right],
$$

для функции $f_{14,8}(s)$ - экспоненту

$$
(-1) w_{8} \Delta_{00} \int_{0}^{\pi} q(t) d t_{a 88} \exp \left(a\left(w_{1}+w_{2}+\cdots+w_{7}+w_{8}\right) s \pi\right)
$$

у функций $f_{14, n}(s)(n=9,10, \ldots, 15)$ для сектора $V_{1}$ таких экспонент нет.

Поэтому справедливо следующее утверждение.

Теорема 3. Уравнение на собственные значения периодической краевой задачи (1.1), (1.2) с условием суммируемости потенциала (1.3) в секторе $V_{1}$ индикаторной диаграммы (5.2) имеет следующий вид:

$$
\begin{aligned}
& g_{1}(s)=(-1) \Delta_{00} \exp \left(a\left(w_{1}+w_{2}+\cdots+w_{7}+w_{8}\right) s \pi\right)-\exp \left(a\left(w_{1}+w_{2}+\cdots+w_{7}\right) s \pi\right) \\
&-\frac{1}{15 a^{14} s^{14}}\left\{\Delta _ { 0 0 } \int _ { 0 } ^ { \pi } q ( t ) d t _ { a 1 1 } \left[\exp \left(a\left(w_{1}+w_{2}+\cdots+w_{7}\right) s \pi\right)\right.\right. \\
&\left.-\exp \left(a\left(w_{1}+w_{2}+\cdots+w_{7}+w_{8}\right) s \pi\right)\right]\left[w_{1}+w_{2}+\cdots+w_{7}\right] \\
&\left.-w_{8} \Delta_{00}\left(\int_{0}^{\pi} \cdots\right)_{a 88} \exp \left(a\left(w_{1}+w_{2}+\cdots+w_{7}+w_{8}\right) s \pi\right)\right\}+\underline{O}\left(\frac{1}{s^{28}}\right)=0 .
\end{aligned}
$$

Учитывая, что из формулы (3.6) следует

$$
\int_{0}^{\pi} q(t) d t_{a 11}=\int_{0}^{\pi} q(t) d t_{a 22}=\cdots=\int_{0}^{\pi} q(t) d t_{a n n} \quad(n=1,2, \ldots, 15),
$$

поделим в уравнении $(6.4)$ на $\left(-\Delta_{00}\right) \exp \left(a\left(w_{1}+w_{2}+\cdots+w_{7}\right) s \pi\right) \neq 0$, получим

$$
\begin{gathered}
g_{1}(s)=\left[e^{a w_{8} s \pi}-1\right]-\frac{1}{15 a^{14} s^{14}} \int_{0}^{\pi} q(t) d t_{a 11}\left\{\left[e^{a w_{8} s \pi}-1\right]\left(w_{1}+w_{2}+\cdots+w_{7}\right)+w_{8} e^{a w_{8} s \pi}\right\} \\
+\underline{O}\left(\frac{1}{s^{28}}\right)=0 .
\end{gathered}
$$

Основное приближение уравнения (6.5) имеет вид $e^{a w_{8} s \pi}-1=0$, откуда получаем

$$
e^{a w_{8} s \pi}=1=e^{2 \pi k i} \Leftrightarrow s_{k, 1, \mathrm{ocH}}=\frac{2 i k}{a w_{8}}, \quad k \in \mathbb{N} .
$$

Это позволяет узнать, в каком виде нужно искать асимптотику корней уравнения (6.5).

Теорема 4. Асимптотика собственных значений дифференииального оператора (1.1)-(1.3) в секторе $V_{1}$ индикаторной диаграммы (5.2) имеет следующий вид:

$$
s_{k, 1}=\frac{2 i}{a w_{8}}\left[k+\frac{d_{14, k, 1}}{k^{14}}+\underline{O}\left(\frac{1}{k^{28}}\right)\right], \quad k \in \mathbb{N} .
$$


Д о к а з а т е л ь с т в о. Для доказательства теоремы 4 необходимо показать, что коэффициенты $d_{14, k, 1}$ формулы (6.6) находятся единственным образом и привести явные формулы для их вычисления. Применяя формулы Маклорена, имеем

$$
\begin{aligned}
& \left.e^{a w_{8} s \pi}\right|_{s_{k, 1}} \stackrel{(6.6)}{=} \exp \left[a w_{8} \pi \frac{2 i}{a w_{8}}\left(k+\frac{d_{14, k, 1}}{k^{14}}+\underline{O}\left(\frac{1}{k^{28}}\right)\right)\right]=1+\frac{2 \pi i d_{14, k, 1}}{k^{14}}+\underline{O}\left(\frac{1}{k^{28}}\right) ; \\
& \left.\frac{1}{s^{14}}\right|_{s_{k, 1}}=\frac{a^{14} w_{8}^{14}}{2^{14} i^{14}} \frac{1}{k^{14}}\left(1+\underline{O}\left(\frac{1}{k^{15}}\right)\right) .
\end{aligned}
$$

Подставляя формулы (6.6), (6.7) в уравнение (6.5) получаем

$$
\begin{gathered}
{\left[1+\frac{2 \pi i d_{14, k, 1}}{k^{14}}+\underline{O}\left(\frac{1}{k^{28}}\right)-1\right]-\frac{1}{15 a^{14}} \frac{a^{14} w_{8}^{14} w_{8}}{2^{14} i^{12} i^{2} w_{8}} \frac{1}{k^{14}}\left(1+\underline{O}\left(\frac{1}{k^{15}}\right)\right)} \\
\times \int_{0}^{\pi} q(t) d t_{a 11}\left\{\left[1+\frac{2 \pi i d_{14, k, 1}}{k^{14}}-1+\underline{O}\left(\frac{1}{k^{28}}\right)\right]\left(w_{1}+w_{2}+\cdots+w_{7}\right)\right. \\
\left.+w_{8}\left[1+\frac{2 \pi i}{k^{14}}+\underline{O}\left(\frac{1}{k^{28}}\right)\right]\right\}+\underline{O}\left(\frac{1}{k^{28}}\right)=0 .
\end{gathered}
$$

Приравнивая в уравнении (6.8) коэффициенты при $k^{-14}$, находим

$$
d_{14, k, 1}=\frac{i}{15 \pi 2^{15}} \int_{0}^{\pi} q(t) d t_{a 11}, \quad k=1,2,3, \ldots
$$

Формула (6.9) показывает, что все коэффициенты $d_{14, k, 1}(k=1,2,3, \ldots)$ из $(6.6)$ определяются единственным образом и вычисляются по формуле (6.9), тем самым теорема 4 доказана.

Изучая аналогичным образом сектор $V_{2}$ индикаторной диаграммы (5.2), мы должны оставить в уравнении (4.5)-(4.12) экспоненты, соответствующие точкам $E_{1}$ и $D_{1}$, т. е. экспоненты $\exp \left(a\left(w_{1}+w_{2}+\cdots+w_{7}+w_{8}\right) s \pi\right)$ и $\exp \left(a\left(w_{2}+w_{3}+\cdots+w_{7}+w_{8}\right) s \pi\right)$.

Произведя рассуждения и выкладки, аналогичные сделанным нами при выводе теорем 3 и 4, приходим к выводу о справедливости следующего утверждения.

Теорема 5. Асимптотика собственных значений дифберенииального оператора (1.1)-(1.3) в секторе $V_{2}$ имеет вид

$$
s_{k, 2}=\frac{2 i}{a w_{1}}\left[k+\frac{d_{14, k, 2}}{k^{14}}+\underline{O}\left(\frac{1}{k^{28}}\right)\right], \quad k \in \mathbb{N},
$$

при этом

$$
d_{14, k, 2}=\frac{i}{15 \pi 2^{15}} \int_{0}^{\pi} q(t) d t_{a 11}, \quad k=1,2,3, \ldots
$$

Изучив аналогичным образом секторы $V_{3}, V_{4}, \ldots, V_{30}$ индикаторной диаграммы (5.2), устанавливаем следующую теорему.

Теорема 6. 1) Асимптотика собственных значений дифференциального оператора (1.1)-(1.3) в секторах $V_{3}, V_{5}, \ldots, V_{29}$ имеет вид

$$
\begin{gathered}
s_{k, 3}=s_{k, 1} e^{\frac{2 \pi i}{15}} ; \quad s_{k, 5}=s_{k, 3} e^{\frac{2 \pi i}{15}}=s_{k, 1} e^{\frac{4 \pi i}{15}} ; \ldots ; \quad s_{k, 2 n-1}=s_{k, 2 n-3} e^{\frac{2 \pi i}{15}}=s_{k, 1} e^{\frac{2 \pi i}{15}(n-1)}, \\
n=1,2, \ldots, 15
\end{gathered}
$$

при этом

$$
\lambda_{k, 2 n-1}=s_{k, 2 n-1}^{15}, \quad n=1,2, \ldots, 15,
$$


величины $s_{k, 1}$ определены формулами (6.6) и (6.9).

2) Асимптотика собственных значений дифференциального оператора (1.1)-(1.3) в секторах $V_{4}, V_{6}, \ldots, V_{30}$ индикаторной диаграммы (5.2) имеет следующий вид:

$$
\begin{gathered}
s_{k, 4}=s_{k, 2} e^{\frac{2 \pi i}{15}} ; \quad s_{k, 6}=s_{k, 4} e^{\frac{2 \pi i}{15}}=s_{k, 2} e^{\frac{4 \pi i}{15}} ; \ldots ; \quad s_{k, 2 n+2}=s_{k, 2 n} e^{\frac{2 \pi i}{15}}=s_{k, 2} e^{\frac{2 \pi i}{15} n}, \\
n=0,1,2, \ldots, 14 ;
\end{gathered}
$$

при этом

$$
\lambda_{k, 2 n+2}=s_{k, 2 n+2}^{15}, \quad n=0,1,2, \ldots, 14, \quad k \in \mathbb{N},
$$

величины $s_{k, 2}$ определены формулами (6.10) и (6.11).

Формулы (6.6)-(6.15) позволяют изучать асимптотику собственных функций дифференциального оператора (1.1)-(1.3) и выписывать формулы регуляризованных следов оператора (1.1), (1.2) в случае условия (1.3) суммируемости потенциала.

\section{СПИСОК ЛИТЕРАТУРЫ}

1. Левитан Б.М., Саргсян И.С. Введение в спектральную теорию. М.: Наука, 1970. 672 с.

2. Марченко В.А. Некоторые вопросы теории одномерных линейных дифференциальных операторов второго порядка // Тр. Моск. мат. общества. 1952. С. 327-420.

3. Лидский В.Б., Садовничий В.А. Асимптотические формулы для корней одного класса целых функций // Мат. сб. 1968. Т. 65, № 4. С. 558-566.

4. Лидский В.В., Садовничий В.А. Регуляризованные суммы корней одного класса целых функций // Функциональный анализ и его приложения. 1967. Т. 1, № 2. С. 52-59.

5. Садовничий В.А. О следах обыкновенных дифференциальных операторов высших порядков // Мат. сб. 1967. Т. 72, № 2. С. 293-310.

6. Бабаджанов Б.А., Хасанов А.Б., Яхшимуратов А.Б. Об обратной задаче для квадратичного пучка операторов Штурма - Лиувилля с периодическим потенциалом // Дифференц. уравнения. 2005. T. 41, № 3. С. 298-305.

7. Федотов А.А., Щетка Е.В. Комплексный метод ВКБ для разностного уравнения Шредингера, потенциал которого - тригонометрический полином // Алгебра и анализ. 2017. Т. 29, № 2. C. $193-219$.

8. Митрохин С.И. О формулах регуляризованных следов для дифференциальных операторов второго порядка с разрывными коэффициентами // Вестн. МГУ. Сер. Математика. Механика. 1986. № 6. C. 3-6.

9. Митрохин С.И. О спектральных свойствах дифференциальных операторов с разрывными коэффициентами // Дифференц. уравнения. 1992. Т. 28, № 3. С. 530-532.

10. Митрохин С.И. О некоторых спектральных свойствах дифференциальных операторов второго порядка с разрывной весовой функцией // Докл. АН. 1997. Т. 356, № 1. С. 13-15.

11. Абдуллаев А.P., Скачкова Е.А. Периодическая краевая задача для дифференциального уравнения четвертого порядка // Изв. вузов. Математика. 2013. №12. С. 3-10.

12. Баданин А.В., Коротяев Е.Л. Спектральные оценки для периодического оператора четвертого порядка // Алгебра и анализ. 2010. Т. 22, № 5. С. 1-48.

13. Поляков Д.М. Спектральный анализ дифференциального оператора четвертого порядка с периодическими и антипериодическими краевыми условиями // Алгебра и анализ. 2015. Т. 27, № 5. C. $117-152$.

14. Поляков Д.М. О спектральных свойствах дифференциального оператора четвертого порядка с периодическими и антипериодическими краевыми условиями // Изв. вузов. Математика. 2015. № 5. С. $75-79$.

15. Винокуров В.А., Садовничий В.А. Асимптотика любого порядка собственных значений и собственных функций краевой задачи Штурма - Лиувилля на отрезке с суммируемым потенциалом // Изв. РАН. Сер. Математика. 2000. Т. 64, № 4. С. 47-108.

16. Митрохин С.И. О спектральных свойствах дифференциального оператора четвертого порядка с суммируемыми коэффициентами // Труды МИАН. 2010. Т. 270. С. 188-197.

17. Митрохин С.И. О спектральных свойствах дифференциальных операторов нечетного порядка с суммируемым потенциалом // Дифференц. уравнения. 2011. Т. 47, № 2. С. 1808-1811. 
18. Митрохин С.И. О спектральных свойствах семейства дифференциальных операторов высокого четного порядка с суммируемым потенциалом // Вест. Волгоград. гос. ун-та. Сер. Физика. Математика. 2016. № 4. С. 121-135.

19. Митрохин С.И. О спектральных свойствах одного дифференциального оператора с суммируемыми коэффициентами с запаздывающим аргументом // Уфим. мат. журн. 2011. Т. 3, № 4. С. $95-115$.

20. Митрохин С.И. Периодическая краевая задача для дифференциального оператора четвертого порядка с суммируемым потенциалом // Владикавказ. мат. журн. 2017. Т. 19, № 4. С. 35-49.

21. Наймарк М.А. Линейные дифференциальные операторы. М.: Наука, 1969. 528 с.

22. Беллман Р., Кук К.Л. Дифференциально-разностные уравнения. М.: Мир, 1967. 548 с.

Поступила 18.09.2018

После доработки 5.11.2018

Принята к публикации 12.11.2018

Митрохин Сергей Иванович

канд. физ.-мат. наук, доцент, профессор РАЕ,

старший науч. сотрудник,

Научно-исследовательский вычислительный центр МГУ им. Ломоносова,

г. Москва

e-mail: mitrokhin-sergey@yandex.ru .

\section{REFERENCES}

1. Levitan B.M., Sargsyan I.S. Introduction to spectral theory: Selfadjoint ordinary differential operators. Translations of Mathematical Monographs, vol. 39, Providence: AMS, 1975, 539 p. ISBN: 082181589X. Original Russian text published in Levitan B.M., Sargsyan I.S. Vvedenie v spektral'nuyu teoriyu. Moscow: Nauka Publ., 1970, 672 p.

2. Marchenko V.A. Some questions of the theory of one-dimensional linear differential operators of the second order. I. Trudy Moskov. Mat. Obshch., 1952, pp. 327-420 (in Russian).

3. Lidskii V.B., Sadovnichii V.A. Asymptotic formulas for the zeros of a class of entire functions. Math. USSR-Sb., 1968, vol. 4, no. 4, pp. 519-527. doi: 10.1070/SM1968v004n04ABEH002812 .

4. Lidskii V.B., Sadovnichii V.A. Regularized sums of zeros of a class of entire functions. Funct. Anal. Appl., 1967, vol. 1, no. 2, pp. 133-139. doi: 10.1007/BF01076085.

5. Sadovnichii V.A. The trace of ordinary differential operators of high order. Math. USSR-Sb., 1967, vol. 1, no. 2, pp. 263-288. doi: 10.1070/SM1967v001n02ABEH001979.

6. Babadzhanov B.A., Khasanov A.B., Yakhshimuratov A.B. On the inverse problem for a quadratic pencil of Sturm-Liouville operators with periodic potential. Differ. Equ., 2005, vol. 41, no. 3, pp. 310-318. doi: $10.1007 / \mathrm{s} 10625-005-0163-9$.

7. Fedotov A.A., Shchetka E.V. Complex WKB method for a difference Schrödinger equation with the potential being a trigonometric polynomial. St. Petersburg Math. J., 2018, vol. 29, no. 2, pp. 363-381. doi: $10.1090 / \mathrm{spmj} / 1497$.

8. Mitrokhin S.I. Formulas for the regularized traces of the second order differential operators with discontinuous coefficients. Mosc. Univ. Math. Bull., 1986, vol. 41, no. 6, pp. 1-5.

9. Mitrokhin S.I. On spectral properties of differential operators with discontinuous coefficients. Differ. Uravn., 1992, vol. 28, no. 3, pp. 530-532 (in Russian).

10. Mitrokhin S.I. On some spectral properties of differential operators of second order with discontinuous positive weight function. Dokl. Akad. Nauk, 1997, vol. 356, no. 1, pp. 13-15.

11. Abdullaev A.R. Skachkova E.A. Periodic boundary-value problem for a fourth-order differential equation. Russian Math. (Iz. VUZ), 2013, vol. 57, no. 12, pp. 1-7. doi: 10.3103/S1066369X13120013.

12. Badanin A.V., Korotyaev E.L. Spectral estimates for a periodic fourth-order operator. St. Petersburg Math. J., 2011, vol. 22, no. 5, pp. 703-736. doi: 10.1090/S1061-0022-2011-01164-1.

13. Polyakov D.M. Spectral analysis of a fourth order differential operator with periodic and antiperiodic boundary conditions. St. Petersburg Math. J., 2016, vol. 27, no. 5, pp. 789-811. doi: 10.1090/spmj/1417.

14. Polyakov D.M. On spectral properties of fourth order differential operator with periodic and semiperiodic boundary conditions. Russian Math. (Iz. VUZ), 2015, vol. 59, no. 5, pp. 64-68. doi: 10.3103/S1066369X15050096 . 
15. Vinokurov V.A., Sadovnichii V.A. Asymptotics of any order for the eigenvalues and eigenfunctions of the Sturm-Liouville boundary-value problem on a segment with a summable potential. Izv. Math., 2000, vol. 64, no. 4, pp. 695-754. doi: 10.1070/IM2000v064n04ABEH000295.

16. Mitrokhin S.I. Spectral properties of a fourth-order differential operator with integrable coefficients. Proc. Steklov Inst. Math., 2010, vol. 270, no. 1, pp. 184-193. doi: 10.1134/S0081543810030144.

17. Mitrokhin S.I. On the spectral properties of odd-order differential operators with integrable potential. Differ. Equ., 2011, vol. 47, no. 12, pp. 1833-1836. doi: 10.1134/S0012266111120123.

18. Mitrokhin S.I. On spectral properties of the family of high even order differential operators with a summable potential. Vestnik Voronezh. Gos. Univ. Ser. Matematika, 2016, no. 4, pp. 121-135 (in Russian).

19. Mitrokhin S.I. On spectral properties of a differential operator with summable coefficients with a retarded argument. Ufimsk. Mat. Zh., 2011, vol. 3, no. 4, pp. 95-115 (in Russian).

20. Mitrokhin S.I. A periodic boundary value problem for a fourth order differential operator with a summable potential. Vladikavkaz. Mat. Zh., 2017, vol. 19, no. 4, pp. 35-49 (in Russian).

21. Naimark M.A. Linear differential operators. Mineola, N.Y.: Dover Publ., 2009, 528 p. ISBN: 9780486468976. Original Russian text published in Naimark M.A., Lineinye differentsial'nye operatory, Moscow: Nauka Publ., 1969, 528 p.

22. Bellman R., Cooke K.L. Differential-difference equations. N Y: Acad. Press, 1963, 462 p. ISBN: 9780080955148. Translated to Russian under the title Differentsial'no-raznostnye uravneniya. Moscow: Mir Publ., 1967, 548 p.

Received September 18, 2018

Revised November 5, 2018

Accepted November 12, 2018

Sergey Ivanovich Mitrokhin, Cand. Sci. (Phys.-Math.), Prof. of the Russian Academy of Natural Sciences, Scientific-Research Computing Center of the Moscow State University (Lomonosov), Moscow, 119991 Russia, e-mail: mitrokhin-sergey@yandex.ru . 